\title{
Cardiac events detection using curvelet transform
}

\author{
ALKA BARHATTE ${ }^{1, *}$, MANISHA DALE ${ }^{2}$ and RAJESH GHONGADE ${ }^{3}$ \\ ${ }^{1}$ Department of Electronics and Telecommunication, AISSMS-Institute of Information Technology, Pune, India \\ ${ }^{2}$ Department of Electronics and Telecommunication, Modern Education Societies College of Engineering, Pune, \\ India \\ ${ }^{3}$ Department of Electronics and Telecommunication, Bharati Vidypeeth Deemed University College of \\ Engineering, Pune, India \\ e-mail: alka.barhatte@mitpune.edu.in; mpdale@mescoepune.org; rbghongade@gmail.com
}

MS received 20 July 2018; revised 16 August 2018; accepted 13 November 2018; published online 1 February 2019

\begin{abstract}
Cardiac event detection is one of the essential steps in cardiac signal processing, analysis and disease diagnosis. Complete morphology of cardiac waves (P-QRS-T) extracted from the location of R-peak is helpful for feature extraction of many applications related to cardiac diseases classification. Therefore cardiac event detection is a prerequisite for reliable cardiac disease diagnosis, and hence it should be robust and timeefficient so that it can be used for real-time signal processing. This work proposes a novel method for R-peak detection using curvelet transform (CT). It demonstrates the use of curvelet energy with an adaptive threshold to estimate the boundaries around R-peak. The exact R-peak locations are then detected from the input signal with the predefined estimated boundaries. The proposed method is evaluated and analysed with all 48 records from the MIT-BIH arrhythmia database. The experimental analysis result yields an average sensitivity of $99.62 \%$, average positive productivity of $99.74 \%$ and average detection error rate of $0.6 \%$. The results obtained have higher than or comparable indices to those in literature. Thus, the proposed system yields high accuracy, low complexity and high processing speed.
\end{abstract}

Keywords. Cardiac event; curvelet transform; curvelet energy; adaptive threshold; R-peak.

\section{Introduction}

Cardiac signals (ECG) are one of the most important sources of diagnostic information. Thus, analysis of cardiac signal contributes a significant role in diagnosing many cardiac diseases like cardiac arrhythmia, cardiac ischemia, heart rate variability, etc. Traditional technologies for cardiovascular diagnoses used at home, clinics and hospitals have remained interested area for researchers to improve them [1]. The cardiac signal is a pseudo-periodic, nonstationary signal that represents the electrical activity of a human heart. For each heartbeat, it lasts roughly $0.8 \mathrm{~s}$ [2]. Every cardiac signal is roughly composed of series of waveforms such as $\mathrm{P}$ wave, QRS complex and $\mathrm{T}$ wave. The QRS complex, which represents ventricle depolarization, is considered to be the most important waveform that leads to the most distinguishing feature of ECG. The peak of this complex is known as R-peak and is the most important point in virtually every ECG algorithm [3]. The frequency of $\mathrm{P}$ and $\mathrm{T}$ waves generally lies between 0.5 and $10 \mathrm{~Hz}$, and that of QRS complex ranges from 4 to $20 \mathrm{~Hz}$ [4]. The diagnostic frequency range for ECG signal is considered to

*For correspondence be $0.05-150 \mathrm{~Hz}$, with signal amplitudes lying between 0.1 and $5 \mathrm{mV}$. The main challenge in R-peak detection is a presence of various types of noise whose frequency overlaps with the frequency range of the ECG signal. Such noises include noise from baseline wander, muscular activities, movement artefacts, power line interference and poor electrode contact $[5,6]$. Frequencies of these noises are as follows. (i) Power line interference has $50 / 60 \mathrm{~Hz}$ with harmonics. It comes from the power line of ECG signal measurement systems despite proper grounding. The $50 / 60 \mathrm{~Hz}$ notch filter can be used for rejecting the powerline interference. (ii) Patients breathing and bad electrodes mainly cause baseline wonder noise. Its frequency range is usually below $0.8 \mathrm{~Hz}$. The high-pass filter (HPF) with cutoff frequency $0.8 \mathrm{~Hz}$ can be used to remove interference by baseline wander. (iii) Muscular activities produce electromyogram (EMG) noise, and it appears as rapid fluctuations that vary faster than ECG waves. Its frequency ranges from dc to $10 \mathrm{kHz}$. The morphological filter for a unit square-wave structuring with the best width of $0.07 \mathrm{~s}$ can be used to remove the interference of EMG noise. (iv) Motion artefacts that result from the motion of the electrode to the patient's skin. It can produce large amplitude signals in ECG, and its duration is about $100-500 \mathrm{~ms}$. The adaptive 
filters can be used to remove the interference of motion artefacts. Mostly, ECG analysis is carried out in two steps. (i) Filtering of an input signal, which uses digital filters like a bandpass filter, notch filters, HPF and adaptive filters. In the last two decades, linear/nonlinear transformations like continuous wavelet transform (CWT), discrete wavelet transform (DWT) [7], and HAAR wavelet transforms are a very popular tool used for denoising of the ECG signals. Empirical mode decomposition (EMD) method is also represented as one of the denoising tools [8, 9]. (ii) Detection of the most significant R-peak, which can be performed using methods like windowing, applying a threshold and Neural Network (NN)-based prediction systems. Two main problems that affect the techniques based on such dual-stage framework are the following: (i) the frequency band of QRS signal is different for different subjects and even for different beats of the same subject and (ii) the overlapping of frequency bands of the noise and QRS complex [10]. Many kinds of literature proposed a wavelet transform as a denoising tool implemented with first-derivative, Hilbert transform and adaptive threshold [3, 11-14].

QRS complexes characterized by steep-slope derivativebased algorithms have frequently been used in the literature [15]. Tomkins [16] proposed a real-time QRS detection algorithm known as the P-T method based on digital analysis of slope, amplitude and width. A special digital BPF is used to reduce false detection of R-peak caused by various types of interferences and achieves an accuracy of 99.3\%. The P-T method appears to be the most common benchmark given that it incorporates several functional techniques, including LPF, HPF, derivative filtering, squaring and windowing for the detection of R-peaks. Meyer et al [17] have combined the P-T method and wavelet to benefit from the strength of both methods, which works in three aspects: (i) first, the prediction of both algorithms always enters in determining the final decision; (ii) secondly, a flexible combination scheme triggered by two parameters allows balancing the influence of two individual algorithms and (iii) third these parameters are estimated in a data-driven way, allowing adoption of the combination scheme to individual sets. Alexandridi et al [18] substituted HAAR wavelet instead of BPF in the P-T method and claimed that it is computationally simpler and its hardware implementation is cheaper than that of BPF. Xue et al [19] used an NN-based adaptive matched filtering for QRS detection. They used an NN adaptive whitening filter to model the lower frequencies of ECG, which are inherently non-linear and non-stationary [19]. Ghaffari et al [20] implemented a mathematics-based QRS detector using CWT. They used the concept of dominant rescaled wavelet coefficients (DRWC) to magnify the QRS and reduce the effect of other R-peaks [20]. Slimane et al [21] proposed QRS detection based on the EMD algorithm. This algorithm requires $\mathrm{HPF}$, signal EMD, a nonlinear transform, integration and finally an LPF [21]. Augustyniak [22] proposed a regression-based QRS detection algorithm that is independent of sampling rate and robust to singular outliers and high-frequency noise. Recently, Sharma and Sharma [15] proposed a technique for QRS detection by pre-processing the ECG signal using weighted total variation (WTV) denoising. The weights are chosen to give preference to preserve QRS complex over $\mathrm{P}$ and $\mathrm{T}$ waves while smoothing, reducing high-frequency noise as well as low-frequency interference from $\mathrm{P}$ and $\mathrm{T}$ waves. CastellsRufas and Carrabina [10] developed a new filter called MaMeMi filter and used it as a nonlinear HPF to remove baseline wander. Wavelet Transform with modified Shannon Energy Envelop is used for rapid ECG analysis $[11,12]$. Ghongade and Ghatol [23] proposed a new technique based on wavelet energy histogram, first-order differentiation, rectification and a fixed threshold. The average detection error rate of the proposed method is found to be $2.26 \%$. According to the author, this system is most suitable for offline signal analysis. Barhatte and Ghongade [24] modified this algorithm by adding Hilbert transform after first-order differentiation block and using adaptive thresholds. The detection error rate obtained is $5.17 \%$.

Literature review shows that the Wavelet Transform is the most commonly used denoising technique for ECG analysis. However, wavelet representation strongly depends upon the choice of the mother wavelet. Therefore, in the case of ECG signals that have varying morphologies, the choice of an appropriate mother wavelet becomes difficult.

A curvelet transform (CT) is a new and effective spectral transform that is becoming popular in denoising of the signals. The CT is organized in such a way that most of the energy of the object is localized in just a few coefficients that can be quantified. Hence, we propose a novel method for QRS complex detection using Fast Discrete Curvelet Transform (FDCT). Here we have calculated the energy of the signal from the curvelet coefficients called curvelet energy, which is used to decide the threshold to locate the boundaries around R-peak and to detect R-peak location.

In section 2, a brief introduction of the CT and curvelet, energy is given. In section 3, the methodology of the proposed system is discussed. In section 4 , the experimental analysis, results and comparison to other state-of-the-art results are shown and discussed. Finally, in section 5, the conclusion is given.

\section{Materials and methods}

\section{$2.1 C T$}

Candès and Donoho proposed the CT in 2000. It is derived from the Ridgelet transform. The CT is a higher-dimensional generalization of the wavelet transform designed to represent complex data at different scales and different angles. In other words, the CT is a multi-scale geometric transform with strong directional character in which 
elements are highly anisotropic at fine scales with effective support shaped according to parabolic scaling principle length $^{2}=$ width [25]. The most important property of curvelets is 'sparsity'. Sparseness is measured by the rate of decay of the $m$-term approximation (reconstruction of the signal using $m$ number of coefficients) of the algorithm. Having a sparse representation offers improved compression possibilities, and also allows for improving denoising performance $[26,27]$.

The curvelet elements can be obtained by parabolic dilation, rotation and translation of specific function $\psi$ [25]. They are indexed by scale parameter $a$, location $b$ and orientation $\theta$. It can be of the form as in Eq. (1):

$$
\psi_{a, b, \Theta}(x)=a^{-3 / 4} \psi\left(\operatorname{DaR}_{\theta}(x-a)\right)
$$

where

$$
D a=\left(\begin{array}{cc}
1 / a & 0 \\
0 & 1 / \sqrt{a}
\end{array}\right),
$$

$D a$ is a parabolic scaling matrix and

$R_{\theta}$ is rotation by $\theta$ radians.

For constructing the curvelet functions, use the following special window functions. Let us consider the scaled Meyer windows (Eq. (2)) [28]:

$$
\begin{gathered}
V(\omega)=\left\{\begin{array}{cc}
1 & |\omega| \leq 1 / 3 \\
\cos \left[\frac{\pi}{2} v(3|\omega|-1)\right] & 1 / 3 \leq|\omega| \leq 2 / 3 \\
0 & \text { else }
\end{array}\right. \\
W(r)=\left\{\begin{array}{cc}
\cos \left[\frac{\pi}{2} v(5-6 r)\right] & 2 / 3 \leq r \leq 5 / 6 \\
1 & 5 / 6 \leq r \leq 4 / 3 \\
\cos \left[\frac{\pi}{2} v(3 r-4)\right] & 4 / 3 \leq r \leq 5 / 3 \\
0 & \text { else }
\end{array}\right.
\end{gathered}
$$

where $v$ is a smoothing function satisfying the condition in Eq. (3):

$$
v(x)=\left\{\begin{array}{ll}
0 & x \leq 0, \\
1 & x \geq 0,
\end{array} \quad v(x)+v(x-1)=1, x \in R .\right.
$$

In practical implementations, one would like to have Cartesian arrays instead of the polar tiling of the frequency plane. Cartesian coronae are based on concentric squares (instead of circles) and shears. Therefore, the construction of window functions on trapezoids instead of polar wedges is desirable. For the transition of the basic curvelet according to the new tiling, where rotation is replaced by shearing [28], we use the form of curvelet with a scale factor of $2^{j}$. The curvelet has useful geometric features that obey a parabolic scaling relation that says that at scale $2^{j}$, each element has an envelope that is aligned along a ridge of length $2^{j / 2}$ and width $2^{j}$ with $j \geq 0$ [29]. Equation (4) represents the form of the curvelet:

$$
\psi_{j, 0,0}(\xi)=2^{-3 j / 4} W\left(2^{-j} \xi_{1}\right) V\left[\frac{2^{|j / 2|} \xi_{2}}{\xi_{1}}\right]
$$

with the window function ' $W$ ' and with a nonnegative window ' $V$ ' with compact support in $[-2 / 3,2 / 3]$.

The CT of function $f(x)$ is given by the convolution integral as follows:

$$
c_{j, l, k}=\left\langle f, \psi_{j, l, k}\right\rangle=\int f(x) \hat{\psi}_{j, 0,0} d x .
$$

\subsection{Curvelet energy}

To calculate curvelet energy, the $\mathrm{CT}$ is applied to a segment of eight samples. The curvelet coefficients obtained for a segment are squared and summed to get the energy of one sample. The same procedure is repeated for the samples in a frame of ECG signal. Curvelet coefficients are obtained once using curvelet as a function parameter at the finest level and secondly wavelet as a function parameter at the finest level. Then the percentage energy is obtained by taking the ratio of these two energies. Figure $1 \mathrm{a}$ and $\mathrm{b}$ shows the corresponding percentage energy obtained for the input ECG signal.

\subsection{Cardiac event (R-peak) detection}

Cardiac event detection of ECG is carried out in four different steps:

1) obtaining curvelet energy for the input ECG,

2) deciding the threshold to extract the Region of Interest (ROI),

3) detecting R-peak location and

4) removal of false R-peaks.

2.3a Deciding threshold: It is clear from figure 1 that energy amplitudes corresponding to QRS complex of the input ECG signal are more significant than the remaining part of the signal. Even the noisy signal in figure $1 \mathrm{~b}$ shows significant peaks for the QRS complex. Also, the energy levels corresponding to QRS complex vary a lot from one subject to other. Setting a fixed threshold for R-peak detection is difficult in such cases. Hence, an adaptive amplitude threshold method is used to decide a fixed threshold of a particular subject. The procedure to decide a threshold is explained further.

The energy curve shown in figure 1 is inverted and normalized as in figure 2, so that the proper thresholds can be sets. Now two thresholds are sets, where lower threshold (T1) is set to $1 \%$ of the maximum amplitude of energy signal and the upper threshold (T2) is set to $20 \%$ of the maximum amplitude of energy signal as in Eq. (6):

$$
T 1=0.01 \max (E), \quad T 2=0.2 \max (E)
$$



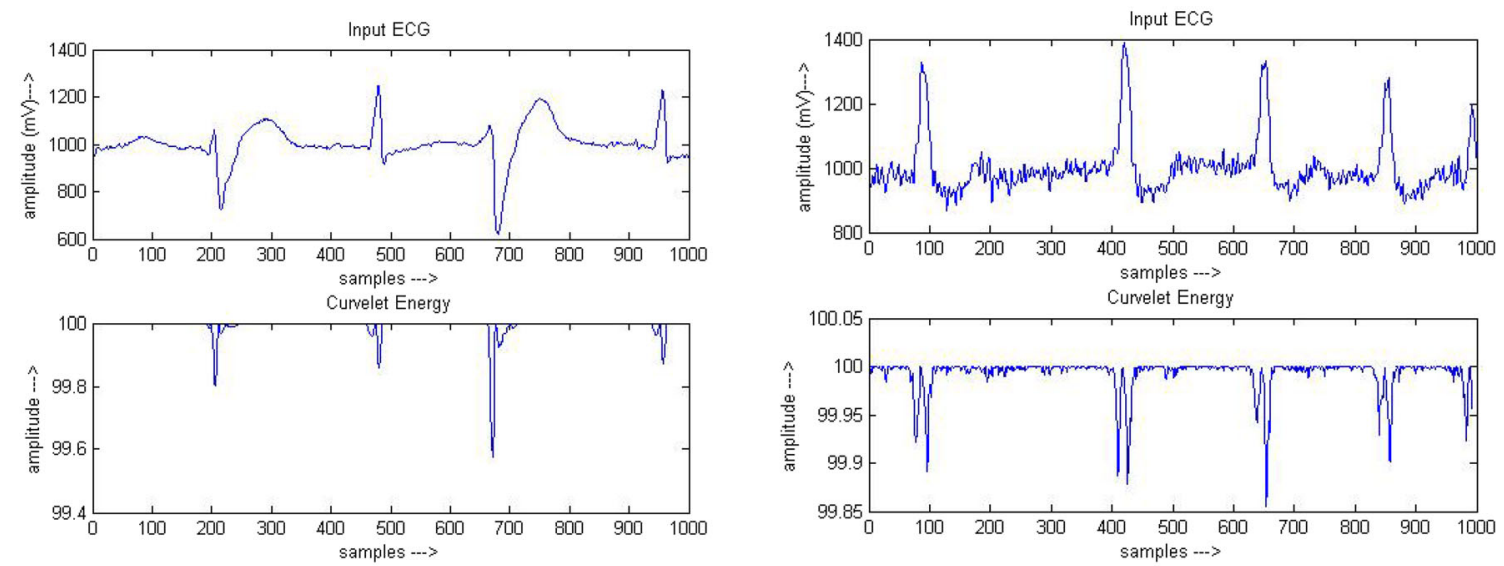

(a) File No.200

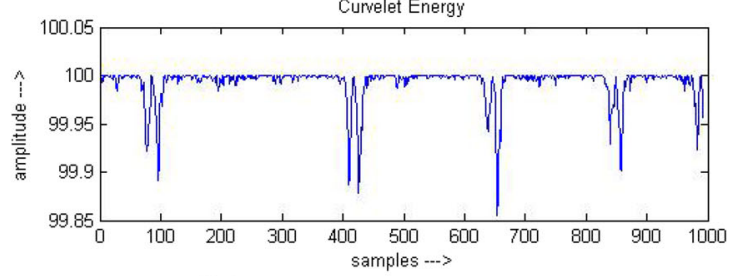

(b) File No. 203(noisy signal)

Figure 1. Curvelet energy plot for ECG.
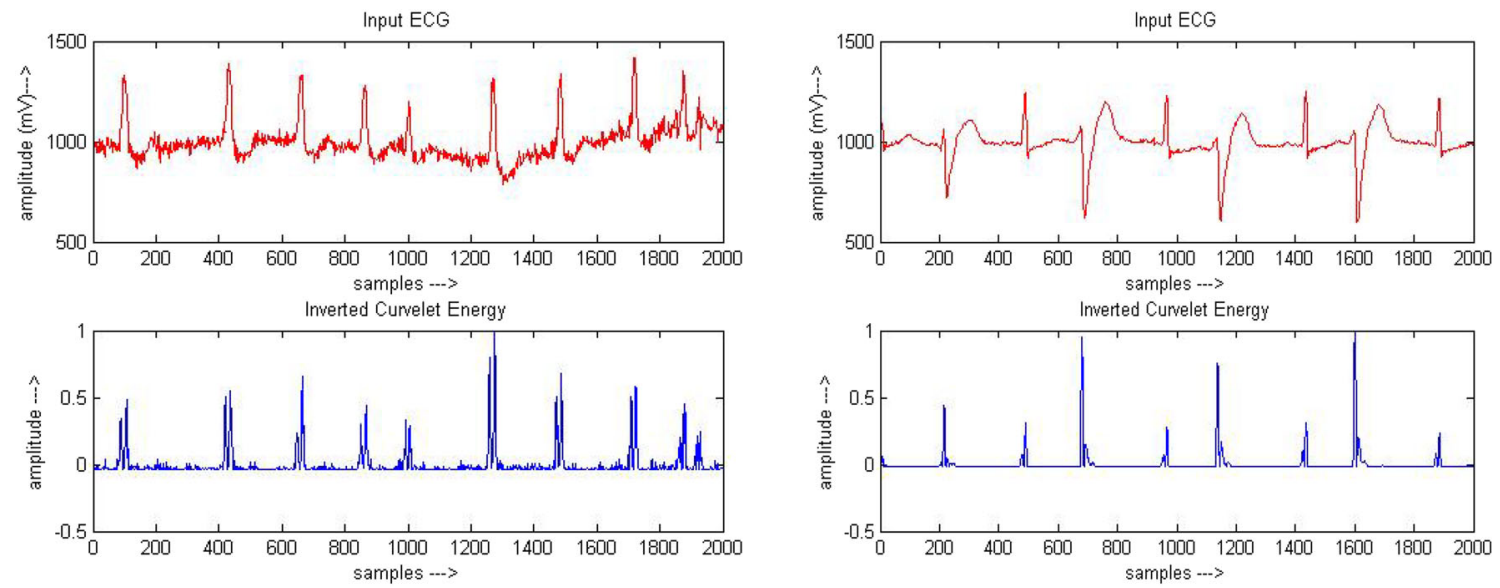

(a) File No 203

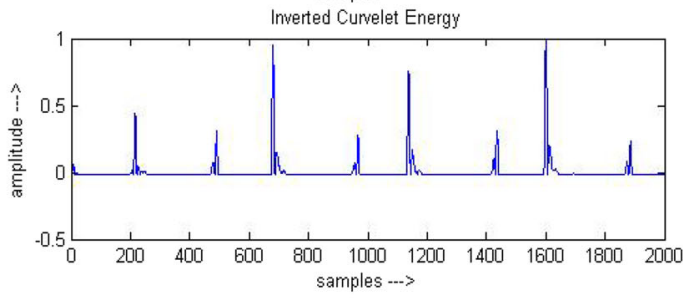

(b) File No 200

Figure 2. Inverted and normalized curvelet energy.

Local maxima counts above this thresholds $T 1$ and $T 2$ are obtained as $N 1$ and $N 2$, respectively. If $N 1$ and $N 2$ are not equal, thresholds are changed according to Eqs. (7) and (8):

$$
\boldsymbol{T} 1=\boldsymbol{T} 1+(\text { delta } \times \boldsymbol{\alpha}), \quad \boldsymbol{T} 2=\boldsymbol{T} 2-(\text { delta } \times \boldsymbol{\alpha})
$$

where delta is the difference between two thresholds and $\alpha$ is a constant of proportionality in which the threshold should increase or decrease.

$$
\text { delta }=T 2-T 1 \text { and } \alpha=0.2 .
$$

The stopping criterion to update the thresholds is $N 1$ becoming equal to $N 2$. At this point, the final threshold $T$ is set to $T 1$. Now, this threshold is used to extract the required ROI of input ECG.

2.3b Deciding ROI: The threshold $T$ obtained from the aforementioned procedure is again compared to the amplitude of energy. If the amplitude of energy is greater than threshold $T$, then set values of a signal (P) as 1 for approximately $0.2 \mathrm{~s}$ for next 70 samples; else set them to zero. The resulting signal from this action is shown in figures 3 and 4 . The ON period of signal (P) gives us required ROI as in figures 3(a) and 4(a). The ROI signal is multiplied by the input ECG signal to extract the region around R-peak as in figures 3(b) and 4(b).

2.3c Detecting R-peak locations: R-peaks can be obtained by detecting maxima of every extracted region around the R-peak. However, it does not work for all the records as R-peaks can be inverted R-peaks in some cases. As shown in figures 4(a) and (b), the input ECG consists of positive $\mathrm{R}$-peaks as well as inverted R-peaks. Hence, the procedure to detect exact R-peak location is explained here.

1. For every extracted R-peak region the minimum and maximum amplitudes are located, and corresponding 

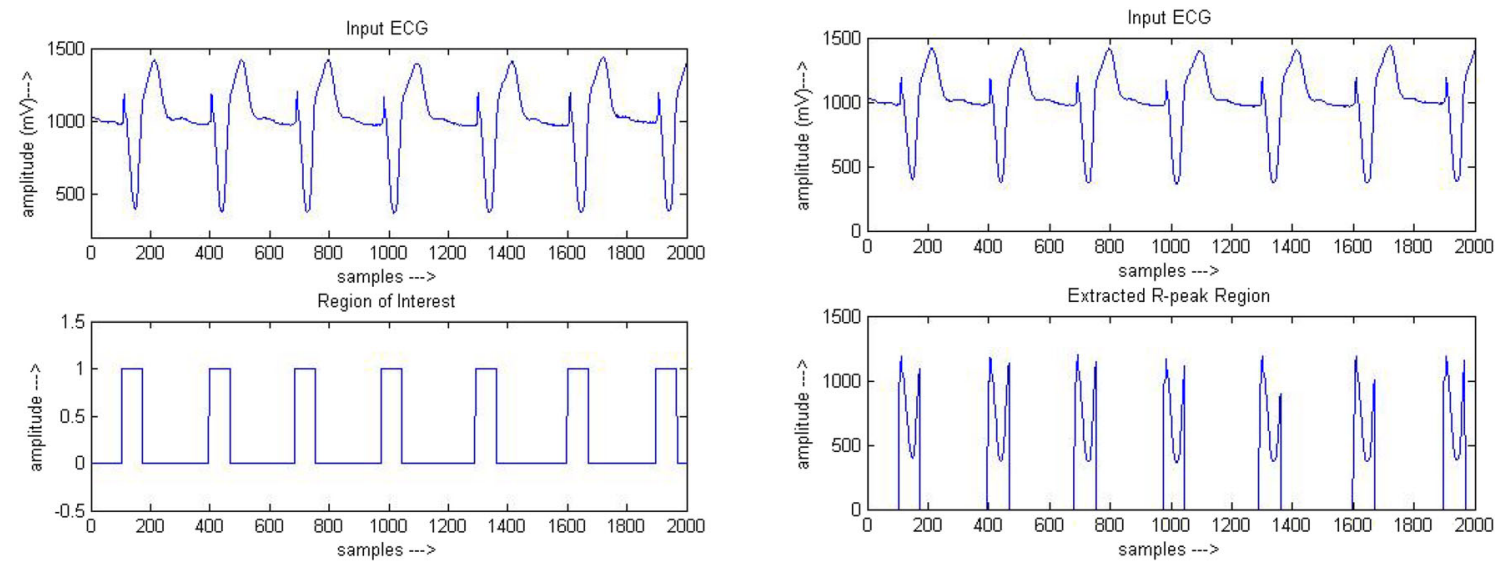

(a) Region of Interest

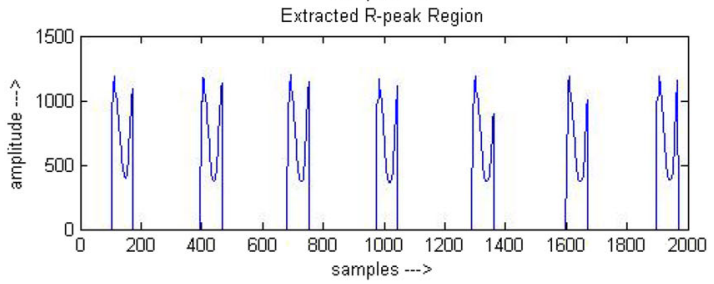

(b) Extracted ROI

Figure 3. Region of interest and extracted ROI for File No. 102.
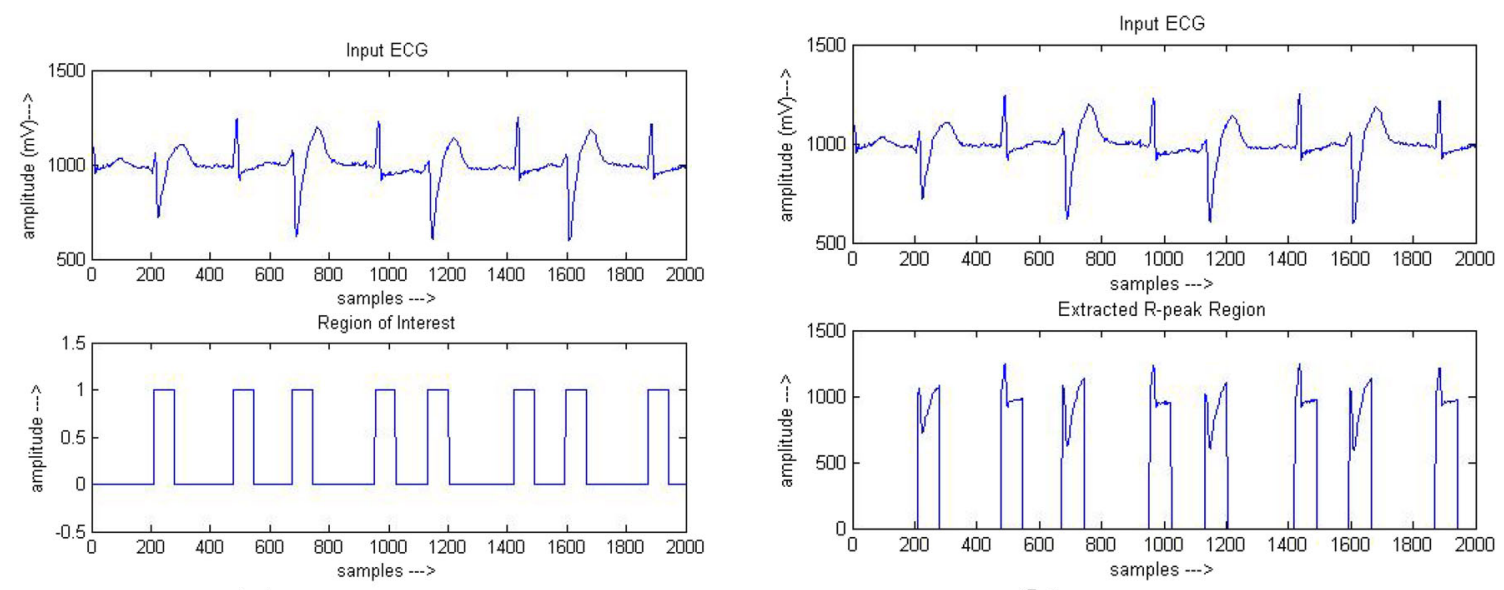

(a) Region of Interest

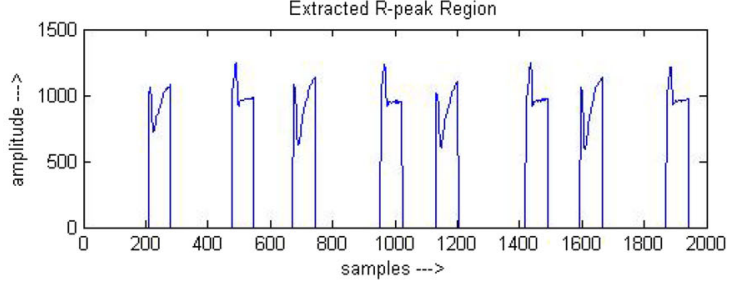

(b) Extracted ROI

Figure 4. Region of interest and extracted ROI for File No. 200.

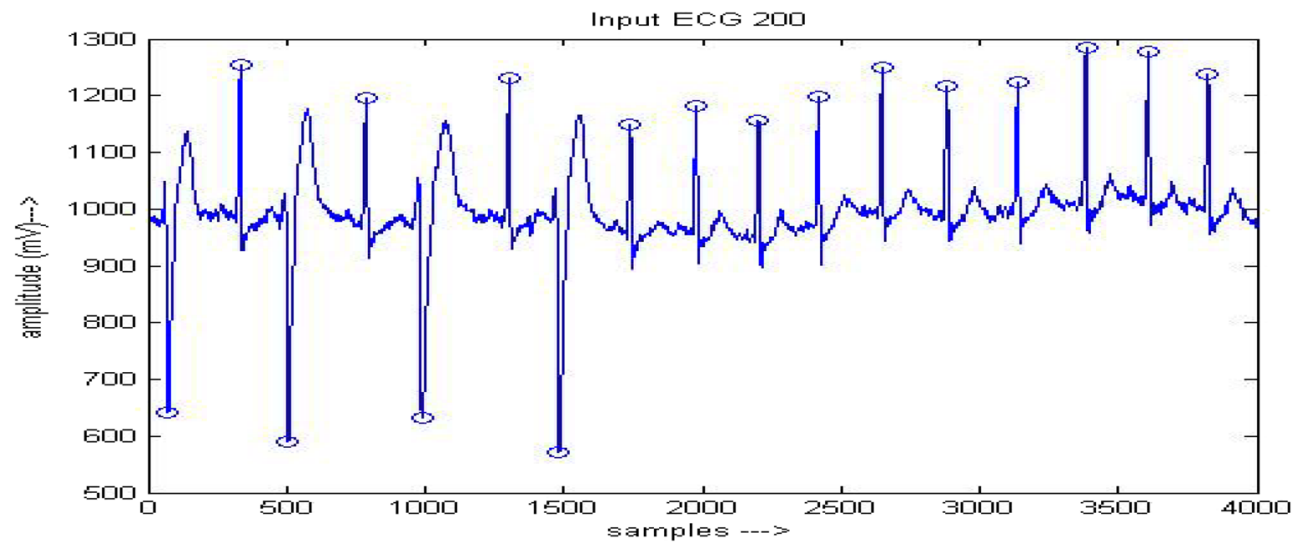

Figure 5. R-peak detection of File No. 200. 

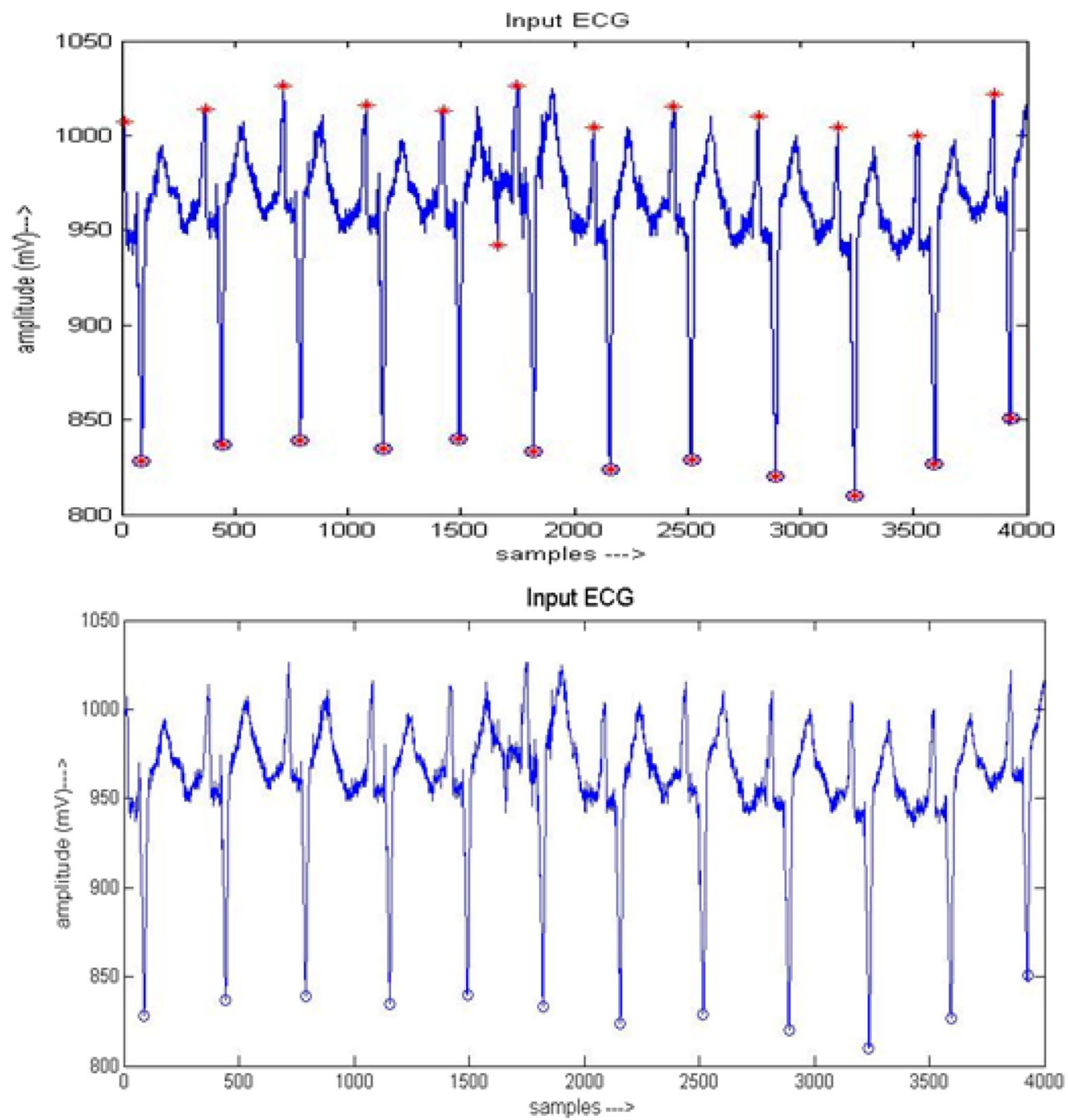

Figure 6. R-peak before and after false R-peak detection.

sample values representing the location of maxima and minima are saved.

2. For deciding the inverted R-peak, the amplitudes of large index location of every extracted R-peak region are compared to the amplitude of the end index location of the corresponding R-peak region.

Let the ROI signal $(p)$ multiplied by input ECG signal (ecg) be represented as extracted R-peak region signal ( $\mathrm{im}$ ), given by Eq. (9):

$$
\operatorname{im}(N)=p(N) \times e c g(N)
$$

where $N$ is the number of samples of input ECG segment.

Obtaining R-peak amplitude locations:

$$
\begin{aligned}
& {[\text { maxloc, } \text { maxval }] \text { of segment }=\max [\operatorname{im}(i) \operatorname{to} i m(i+69)]} \\
& {[\text { minloc, } \text { minval }] \text { of segment }=\min [\operatorname{im}(i) \operatorname{to} i m(i+69)]}
\end{aligned}
$$

where $i$ is a starting point of every ROI segment of signal im.
Now if $($ ecg $($ maxval $)-\operatorname{ecg}(i+69)>30) \quad$ then $R-$ peak location is maxloc of segment

\section{else $\mathrm{R}$ - peak location is minloc of segment.}

Figure 5 shows the R-peak detection of record no. 200 where ' $\mathrm{o}$ ' represents the location of detected R-peak.

2.3d Removal of false R-peak detection: In some cases, $\mathrm{P}$ and $\mathrm{T}$ peaks get enhanced as compared with the normal ECG signal. Hence, energies corresponding to those peaks are also enhanced and it may trigger false detection of R-peak.

The two parameters used to avoid the false R-peak detection are (i) time duration between R-R-peak and (ii) amplitudes of the R-R-peaks.

If two consecutive R-peaks get detected within $0.3 \mathrm{~s}$ of time duration, that is, if the difference between two consecutive R-peak locations is less than 110 samples, then the corresponding R-peak amplitudes are checked. The R-peak 

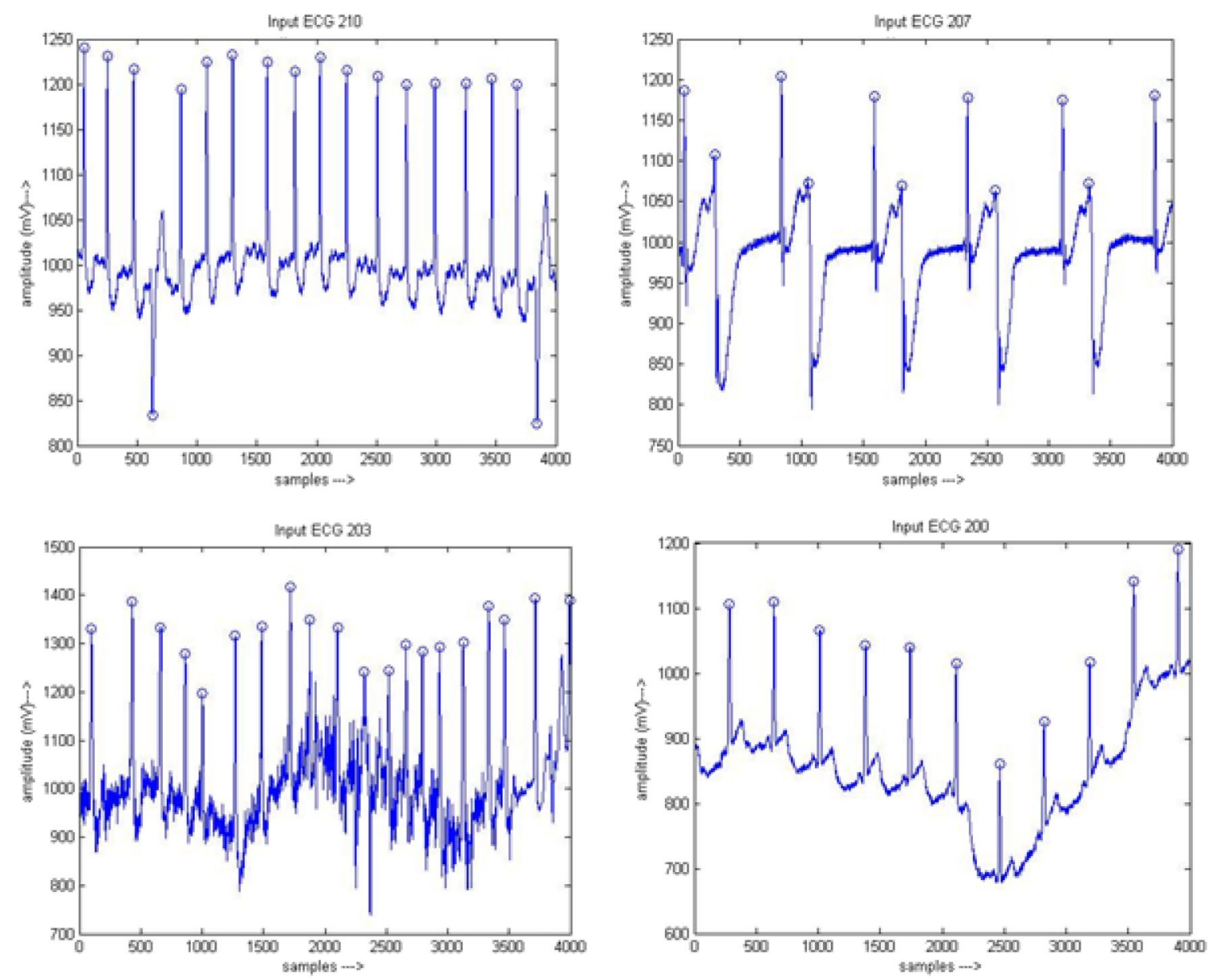

Figure 7. R-peak detection for different records.

corresponding to higher amplitude is the true R-peak, and another one is discarded. This procedure continues for all detected beats.

$$
\begin{array}{r}
\text { for } \boldsymbol{i}=1 \text { to } N-1 \\
\text { if }(\boldsymbol{r p i n d}(\boldsymbol{i})-\boldsymbol{r p i n d}(\boldsymbol{i}+1)<110)
\end{array}
$$

then check for amplitudes corresponding to

two index values as

$\operatorname{if}(\operatorname{abs}(\operatorname{ecg}(\boldsymbol{r p i n d}(\boldsymbol{i})))>\operatorname{abs}(\boldsymbol{e c g}(\boldsymbol{r p i n d}(\boldsymbol{i}+1))))$

then shift all the $R$ - peaks to the left by one position

from $i+2$ toN

else shift all the $R$ - peaks to the left by one position

from $\mathrm{i}+1$ to $\mathrm{N}$

and reduce $N$ to $N-1$ for every satisfied condition

The record in figure 6 has higher R-peaks amplitude and inverted R-peaks. The star points in figure 6 show the R-peak locations detected before removal of false $\mathrm{R}$-peak and the second figure shows the true R-peaks detections after removal of the false R-peak algorithm.

\section{Results and analysis}

Evaluation and validation of the proposed algorithm are performed using the MIT-BIH Arrhythmia database. It consists of 48 recordings with duration of $30 \mathrm{~min}$ each. The sampling frequency of these recordings is $360 \mathrm{~Hz}$ with 11 bits over $10 \mathrm{mV}$ range. Two cardiologists annotate all records. The algorithm is implemented on a $2.4-\mathrm{GHz}$ Intel core $\mathrm{i} 3.3110 \mathrm{M}$ processor using MATLAB version 14 . Detected R-peaks are compared to the annotations of $\mathrm{R}$ points from the MIT-BIH database.

Figure 7 shows the output of R-peak detection for different morphologies of QRS complexes.

Table 1 summarizes the result of R-peak detection.

The evaluation of the proposed algorithm is performed based on the following three performance parameters: sensitivity, positive predictivity and detection error rate. After evaluating the proposed algorithm on the above metrics the results obtained are average sensitivity of $99.64 \%$, average positive predictivity of 99.74 and the average detection error rate is $0.6 \%$. Table 2 compares the results obtained using the proposed method with the 
Table 1. The result of R-peak detection on MIT-BIH database.

\begin{tabular}{|c|c|c|c|c|c|c|}
\hline $\begin{array}{l}\text { Record } \\
\text { no. }\end{array}$ & $\begin{array}{l}\text { Total } \\
\text { no. of } \\
\text { beats }\end{array}$ & $\mathrm{FP}$ & $\mathrm{FN}$ & $\begin{array}{c}\text { Positive } \\
\text { predictivity }\end{array}$ & Sensitivity & DER \\
\hline 100 & 2273 & 0 & 0 & 1.0000 & 1.0000 & 0.0000 \\
\hline 101 & 1865 & 1 & 0 & 0.9995 & 1.0000 & 0.0005 \\
\hline 102 & 2187 & 41 & 41 & 0.9813 & 0.9813 & 0.0375 \\
\hline 103 & 2084 & 0 & 2 & 0.0000 & 0.9990 & 0.0010 \\
\hline 104 & 2229 & 31 & 5 & 0.9862 & 0.9978 & 0.0162 \\
\hline 105 & 2572 & 33 & 12 & 0.9873 & 0.9953 & 0.0175 \\
\hline 106 & 2027 & 2 & 7 & 0.9990 & 0.9965 & 0.0044 \\
\hline 107 & 2137 & 8 & 0 & 0.9963 & 1.0000 & 0.0037 \\
\hline 108 & 1763 & 73 & 63 & 0.9588 & 0.9643 & 0.0771 \\
\hline 109 & 2532 & 0 & 1 & 1.0000 & 0.9996 & 0.0004 \\
\hline 111 & 2124 & 0 & 4 & 1.0000 & 0.9981 & 0.0019 \\
\hline 112 & 2539 & 0 & 0 & 1.0000 & 1.0000 & 0.0000 \\
\hline 113 & 1795 & 0 & 0 & 1.0000 & 1.0000 & 0.0000 \\
\hline 114 & 1879 & 0 & 1 & 1.0000 & 0.9995 & 0.0005 \\
\hline 115 & 1953 & 0 & 1 & 1.0000 & 0.9995 & 0.0005 \\
\hline 116 & 2412 & 0 & 24 & 1.0000 & 0.9900 & 0.0100 \\
\hline 117 & 1535 & 0 & 0 & 1.0000 & 1.0000 & 0.0000 \\
\hline 118 & 2278 & 0 & 0 & 1.0000 & 1.0000 & 0.0000 \\
\hline 119 & 1987 & 6 & 0 & 0.9970 & 1.0000 & 0.0030 \\
\hline 121 & 1863 & 2 & 0 & 0.9989 & 1.0000 & 0.0011 \\
\hline 122 & 2476 & 0 & 0 & 1.0000 & 1.0000 & 0.0000 \\
\hline 123 & 1518 & 0 & 3 & 1.0000 & 0.9980 & 0.0020 \\
\hline 124 & 1619 & 0 & 0 & 1.0000 & 1.0000 & 0.0000 \\
\hline 200 & 2601 & 0 & 2 & 1.0000 & 0.9992 & 0.0008 \\
\hline 201 & 1963 & 0 & 5 & 1.0000 & 0.9975 & 0.0025 \\
\hline 202 & 2136 & 0 & 5 & 1.0000 & 0.9977 & 0.0023 \\
\hline 203 & 2980 & 27 & 80 & 0.9908 & 0.9732 & 0.0359 \\
\hline 205 & 2656 & 0 & 13 & 1.0000 & 0.9951 & 0.0049 \\
\hline 207 & 1860 & 22 & 19 & 0.9882 & 0.9898 & 0.0220 \\
\hline 208 & 2955 & 2 & 20 & 0.9993 & 0.9932 & 0.0074 \\
\hline 209 & 3005 & 1 & 0 & 0.9997 & 1.0000 & 0.0003 \\
\hline 210 & 2650 & 14 & 6 & 0.9947 & 0.9977 & 0.0075 \\
\hline 212 & 2748 & 0 & 0 & 1.0000 & 1.0000 & 0.0000 \\
\hline 213 & 3251 & 0 & 0 & 1.0000 & 1.0000 & 0.0000 \\
\hline 214 & 2262 & 2 & 7 & 0.9991 & 0.9969 & 0.0040 \\
\hline 215 & 3363 & 0 & 7 & 1.0000 & 0.9979 & 0.0021 \\
\hline 217 & 2208 & 0 & 7 & 1.0000 & 0.9968 & 0.0032 \\
\hline 219 & 2154 & 0 & 3 & 1.0000 & 0.9986 & 0.0014 \\
\hline 220 & 2048 & 0 & 0 & 1.0000 & 1.0000 & 0.0000 \\
\hline 221 & 2427 & 0 & 2 & 1.0000 & 0.9992 & 0.0008 \\
\hline 222 & 2483 & 0 & 0 & 1.0000 & 1.0000 & 0.0000 \\
\hline 223 & 2605 & 0 & 4 & 1.0000 & 0.9985 & 0.0015 \\
\hline 228 & 2053 & 16 & 24 & 0.9922 & 0.9883 & 0.0195 \\
\hline 230 & 2256 & 0 & 0 & 1.0000 & 1.0000 & 0.0000 \\
\hline 231 & 1571 & 2 & 0 & 0.9987 & 1.0000 & 0.0013 \\
\hline 232 & 1780 & 0 & 1 & 1.0000 & 0.9994 & 0.0006 \\
\hline 233 & 3079 & 0 & 24 & 1.0000 & 0.9922 & 0.0078 \\
\hline 234 & 2753 & 0 & 2 & 1.0000 & 0.9993 & 0.0007 \\
\hline Total & 109494 & 283 & 395 & 0.9974 & .9964 & 0.0063 \\
\hline
\end{tabular}

algorithms implemented by authors in previous papers $[23,24]$ and a few existing algorithms.

Table 2 clearly shows the achievable improvement in sensitivity and positive predictivity as compared with our
Table 2. Comparison of various methods with the proposed method.

\begin{tabular}{|c|c|c|c|c|}
\hline References & Method & $\begin{array}{l}\mathrm{SE} \\
(\%)\end{array}$ & $\begin{array}{l}\mathrm{PP} \\
(\%)\end{array}$ & $\begin{array}{c}\text { DER } \\
(\%)\end{array}$ \\
\hline $\begin{array}{l}\text { Proposed } \\
\text { method }\end{array}$ & $\begin{array}{l}\text { Curvelet energy }+ \\
\text { adaptive threshold }\end{array}$ & 99.64 & 99.74 & 0.63 \\
\hline $\begin{array}{l}\text { Ghongade } \\
\text { and Ghatol } \\
\text { [23] }\end{array}$ & $\begin{array}{l}\text { Wavelet energy with a } \\
\text { fixed threshold }\end{array}$ & 98.94 & 98.79 & 2.26 \\
\hline $\begin{array}{l}\text { Barhatte and } \\
\text { Ghongade } \\
\text { [24] }\end{array}$ & $\begin{array}{c}\text { Wavelet energy }+ \text { Hilbert } \\
\text { transform }+ \text { adaptive } \\
\text { thresholds }\end{array}$ & 97.45 & 97.40 & 5.17 \\
\hline $\begin{array}{l}\text { Sharma and } \\
\text { Sharma } \\
\text { [15] }\end{array}$ & $\begin{array}{l}\text { Weighted total variation } \\
\text { denoising }\end{array}$ & 99.90 & 99.88 & 0.22 \\
\hline $\begin{array}{l}\text { Park et al } \\
\quad[11]\end{array}$ & $\begin{array}{c}\text { Wavelet transform and } \\
\text { modified Shannon } \\
\text { energy envelope }\end{array}$ & 99.93 & 99.91 & 0.16 \\
\hline $\begin{array}{l}\text { Castels- } \\
\text { Rufas and } \\
\text { Carrabina } \\
{[10]}\end{array}$ & MaMeMi filter & 99.43 & 99.67 & 0.79 \\
\hline $\begin{array}{l}\text { Leong et al } \\
\text { [30] }\end{array}$ & $\begin{array}{c}\text { Quadratic spline wavelet } \\
\text { transform }\end{array}$ & 99.30 & 99.70 & - \\
\hline $\begin{array}{l}\text { Martinez } \\
\quad \text { et al [12] }\end{array}$ & $\begin{array}{c}\text { A wavelet-based ECG } \\
\text { delineator }\end{array}$ & 99.66 & 99.56 & 0.78 \\
\hline $\begin{array}{l}\text { Tompkins } \\
\text { [16] }\end{array}$ & $\begin{array}{c}\text { First derivative followed } \\
\text { by squaring, windowing } \\
\text { and threshold }\end{array}$ & 99.54 & 99.75 & 0.71 \\
\hline
\end{tabular}

Table 3. Buffer length calculations for different heart rates.

\begin{tabular}{|c|c|c|c|}
\hline Arrhythmias & $\begin{array}{l}\text { Heart rate (beats/ } \\
\text { min) }\end{array}$ & $\begin{array}{l}\text { Time required for } \\
\text { the occurrence of } \\
\text { the next heartbeat } \\
(\mathrm{ms})\end{array}$ & $\begin{array}{c}\text { Buffer } \\
\text { length } \\
(\mathrm{ms})\end{array}$ \\
\hline $\begin{array}{l}\text { Normal } \\
\text { sinus } \\
\text { rhythm }\end{array}$ & $\begin{array}{c}\text { 60-100 (assuming } \\
100)\end{array}$ & 600 & 390 \\
\hline Tachycardia & $\begin{array}{c}>100 \text { (assuming } \\
180)\end{array}$ & 330 & 120 \\
\hline Bradycardia & $\begin{array}{c}<60 \text { (assuming } \\
40 \text { ) }\end{array}$ & 150 & 129 \\
\hline $\begin{array}{l}\text { Average of } \\
\text { three } \\
\text { cases }\end{array}$ & $\begin{array}{c}(100+180+40) / \\
3=107\end{array}$ & 560 & 350 \\
\hline
\end{tabular}

previously proposed methods. Secondly, compared with state-of-the-art results the detection accuracy obtained using the proposed algorithm is good.

The average time required for processing a 30-min record is less than $8 \mathrm{~min}$; that is, the average time required for a single-beat detection is $210 \mathrm{~ms}$. Table 3 shows the time of occurrence required for the next heartbeat for three different types of arrhythmias broadly classified according to their variation in the heartbeat as normal sinus rhythm, 
tachycardia and bradycardia. Then the buffer length is calculated assuming the worst case heart rate of each type. It shows that the minimum overlapping window/buffer length obtained is $120 \mathrm{~ms}$ whereas the average overlapping window/buffer length is $350 \mathrm{~ms}$. It is clear that $210 \mathrm{~ms}$ is sufficient to process the next beat without loss of any information.

\section{Discussion}

The following points contribute to the increase in accuracy of the R-peak-

1. As QRS complexes are usually $0.06-0.12 \mathrm{~s}$ wide (21-44 samples) [10], the width of ROI is considered as 70 samples. The ROI consisting of ON pulse of 70 samples gives the probable locations of R-peak.

2. The positive R-peak can be clearly differentiated from inverted R-peak simply by comparing the amplitudes as explained in section 2.3.2. From figures 6 and 7, it is obvious that if the R-peaks are inverted the higher amplitude is very close to the end of the pulse. Thus, giving the difference of amplitudes of large index and end index locations as almost zero for the safer side, it is chosen as 30

3. Lastly, considering that the maximum heart rate possible is around 180 beats $/ \mathrm{min}$, there should not lay two R-peaks within a range of $0.3 \mathrm{~s}$. Hence the 0.3 -s time duration is used to remove the false R-peaks occurring before $0.3 \mathrm{~s}$. Here again, the amplitudes of two R-peaks detected within the $0.3 \mathrm{~s}$ are used to decide the true $\mathrm{R}$-peak. R-peak with higher amplitude is considered as the true R-peak whereas amplitude with smaller R-peak is considered as noise.

Finally, we would like to mention that the CT and curvelet energy proposed for R-peak detection are the two contributing points in this paper apart from the three points discussed earlier. As we have not used any addition classical filters for pre-processing of ECG signal, the results obtained are comparable to those from other methods. Hence, we can say that CT can be used for denoising of a signal by enhancing the energies of the QRS complex by reducing the effect of noise in the signal.

\section{Conclusion}

In this paper, the authors presented and validated R-peak detection using $\mathrm{CT}$ and the adaptive threshold. The experimental analysis gives an average sensitivity of $99.64 \%$ and average positive predictivity of $99.74 \%$ with a detection rate of $0.6 \%$. The results are compared to the results published in the literature and have shown a reliable, robust and accurate method for R-peak detection. As the average time required for a single-beat detection is $210 \mathrm{~ms}$, which results in the minimum buffer length of $120 \mathrm{~ms}$, the proposed algorithm can be used for online as well as offline R-peak detection.

\section{References}

[1] Barhatte A and Ghongade R 2015 A multiclass cardiac events classifier using clustering and modified adaptive neuro-fuzzy inference system. In: Proceedings of the IEEE International Conference (ICRCICN), https://doi.org/10. 1109/icrcicn.2015.7434216, pp. 90-95

[2] Nehla D and Sadok A 2012 Algebraic approach for R-peak detection in the electrocardiogram (ECG) signal. IEEExplore, https://doi.org/10.1109/iconscs.2012.6502461

[3] Madeiro P, Cortez C, Marques A, Seisdedos R and Sobrinho R 2012 An innovative approach of QRS segmentation based on first-derivative, Hilbert and Wavelet Transforms. Med. Eng. Phys. 34: 1236-1246

[4] Kannathal N, Acharya U, Joseph P, LimChoo M, and Suri J 2007 Analysis of electrocardiogram. Adv. Card. Signal Process. ISBN: 978-540-36674-4

[5] Rodriguez R, Mexicano A, Bila J, Cervantes J and Ponce P 2015 Feature extraction of electrocardiogram signals by applying adaptive threshold and principal component analysis. J. Appl. Res. Technol. 13: 261-269

[6] Raul A, Arturo J M P and Anton Vila Sobrino X 2013 A comparison of three QRS detection algorithms over a public database. Proc. Technol. 9: 1159-1165

[7] Kadambe S, Murray R, and Faye Boudreaux-Bartels G 1999 Wavelet transform-based QRS complex detector. IEEE Trans. Biomed. Eng. 46(7): 838-848

[8] Eduardo P, Octavian P and Pedro G 2012 Empirical Mode Decomposition and Principal Component Analysis implementation in processing non-invasive cardiovascular signal. Measurement 45: 175-181

[9] Md. Kabir A and Shahnaz C 2012 Denoising of ECG signals based on noise reduction algorithms in EMD and wavelet domains. Biomed. Signal Process. Control 7(5): 481-489

[10] Castells-Rufas D and Carrabina J 2015 Simple real-time QRS detector with MaMeMi filter. Biomed. Signal Process. Control 21: 137-145

[11] Park J S, Lee S W and Park U 2017 R peak detection method using WT and modified Shannon energy envelope. $J$. Healthc. Eng. Article ID 4901017

[12] Martinez J P, Almedia R, Salvador O, Rocha A and Laguna P A 2004 Wavelet-based ECG delineator: evaluation on standard databases. IEEE Trans. Biomed. Eng. 51(4): 570-581

[13] Rabbani H, Mahjoobl M P, Farahabadi E and Farahabadi A 2011 R-peak detection in electrocardiogram signal based on an optimal combination of wavelet transform, Hilbert transform, and adaptive thresholding. J. Med. Signals Sens. 1(2): 91-98

[14] Christov I I 2004 Real time electrocardiogram QRS detection using combined adaptive threshold. Biomed. Eng. Online 3: 28

[15] Sharma T and Sharma K K 2017 QRS complex detection in ECG signals using locally adaptive weighted total variation denoising. Comput. Biol. Med. 87: 187-199 
[16] Tompkins W J 1985 A real time QRS detection algorithm. IEEE Trans. Biomed. Eng. BME-32: 230-236

[17] Meyer C, Gavela J F and Harris M 2006 Combining algorithms in automatic detection of QRS complexes in ECG signals. IEEE Trans. Inf. Technol. Biomed. 10(3): 468-475

[18] Alexandridi A, Panagopoulos I, Manis G and Papakonstantinou G 2003 R-peak detection with alternative HAAR wavelet filter. In: Proceedings of the third IEEE International Symposium on Signal Processing and Information Technology, pp. 219-222

[19] Xue Q, Hu Y M and Tompkins W J 1992 Neural-networkbased adaptive matched filtering for QRS detection. IEEE Trans. Biomed. Eng. 39(4): 317-329

[20] Ghaffari A, Golbayani H and Ghasemi M 2008 A new mathematical based QRS detector using continuous wavelet transform. Comput. Electr. Eng. 34: 81-91

[21] Slimane Z E H and Amine N 2010 QRS complex detection using Empirical Mode Decomposition. Digit. Signal Process. 20: $1221-1228$

[22] Augustyniak P 2015 A robust heartbeat detector on ECG sampling rate. In: Proceedings of the International Conference of the IEEE Engineering in Medicine and Biology Society (EMBC), https://doi.org/10.1109/embc.2015.
[23] Ghongade R and Ghatol A 2009 A novel QRS detection method. Int. J. Appl. Comput. 2(Supp. 1): 6-13

[24] Barhatte A and Ghongade R 2014 R-peak detection using wavelet-energy histogram and adaptive thresholding. Int. J. Emerg. Trends Electr. Electron. 10: 141-144

[25] Candès E J 2000 What is a curvelet? Not. AMS 50(11): 1402-1403

[26] Candès E J and Donoho D L 1999 Curvelets—a surprisingly effective nonadaptive representation for objects with edges. In: Curve and surface fitting. Nashville, TN: Vanderbilt University Press

[27] Candès E J and Demanet L 2003 Curvelets, and Fourier integral operators. Compt. Rendus Math. 336: 395-398

[28] Jianwei M and Plonka G 2010 The curvelet transform: a review of recent application. IEEE Signal Process. Mag. 118-133

[29] Candès E J, Demanet L, Donoho D and Ying L 2006 Fast discrete curvelet transform. SIAM Multiscale Model. Simul. 5: 861-899

[30] Leong C, Mak P, Lam C, Dong C, Vai M, Mak P, Pun S, Wan F and Martins R 2012 A $0.83-\mu \mathrm{W}$ QRS detection processor using quadratic spline wavelet transform for wireless ECG acquisition in 0.35- $\mu \mathrm{m}$ CMOS. IEEE Trans. Biomed. Circuits Syst. 6(6): 586-595 